\title{
DrogengebraucherInnen und Drogenhilfe im Justizvollzug - eine Übersicht
}

\author{
Drug Users And Drug Services in Prison - An Overview
}

\section{Zusammenfassung}

Etwa 20\% aller Gefangenen in Deutschlands Strafhaftanstalten weisen einen problematischen Drogengebrauch auf, d.h., dass ca. 15000 Inhaftierte trotz scharfer Kontrollen entweder gegenwärtig noch (mit unterschiedlichen Konsummustern und -frequenzen) oder bis vor kurzem KonsumentIn und/oder HändlerIn illegaler und hier vor allem intravenös applizierter Drogen waren. Diese europaweit zu beobachtende Zunahme der Anzahl von Drogengebrauchern in Haft stellt die medizinische und psychosoziale Versorgung im Vollzug vor große Probleme: Zwischen Krankheit und Kriminalität, Hilfe und Kontrolle schwanken die drogenhelferischen Konzepte. Insbesondere die Verbreitung viraler Infektionskrankheiten (HIV/AIDS und Hepatitiden $B+C$ ) hat die eindimensionale Ausrichtung der Hilfeangebote auf Abstinenz infrage gestellt: Müssen nicht auch im Gefängnis zusätzlich schadensminimierende Angebote entwickelt werden, die sich in Freiheit bewährt haben?

Der Beitrag gibt einen Überblick über die Suchtkrankenhilfeversorgung in der totalen Institution Gefängnis und bietet Perspektiven für eine Umorientierung an.

\section{Schliuisselwörter}

Drogengebrauch · Gefängnisse · Drogenhilfe · Infektionskrankheiten · Prävention

\section{Abstract}

Approximately $20 \%$ of all inmates in German prisons show a problematic pattern of illegal drug use. This means, that despite sharp controls, about 15,000 inmates in German penitentiaries either currently use drugs (with varying patterns and frequencies of use) or have been consumers or traffickers of illegal and mainly injectable drugs shortly before their admission to prison. This increase in the number of incarcerated drug users can be noticed in the whole of Europe. This development is challenging the medical and psycho-social care in prisons. Drug services being offered vary between concepts of illness and criminality, help and control. Particularly the spread of viral infections (like HIV/ AIDS and Hepatitis B and C) is questioning the one-dimensional, abstinence-oriented target of drug services. The question arising from that is: should prisons provide the same harm-reduction services, which have been proven successfully in the community? This contribution gives an overview of the provision of drug services in the total institution prison and offers perspectives for a basic different orientation.

\section{Key words}

Drug use · prisons · drug services · infectious diseases · prevention

\section{Einleitung}

Das Gefängnis bildet spiegelbildlich gesellschaftliche Probleme und individuelle Notlagen ab und muss im Rahmen seiner Möglichkeiten darauf reagieren - auch auf die Verbreitung des Konsums legaler und illegaler Drogen mit all seinen sozialen und ge- sundheitlichen Folgeerscheinungen. Der folgende Beitrag gibt einen Überblick über die Widersprüche in der gesundheitlichen Versorgung Drogenabhängiger im Gefängnis. Im Spannungsfeld von Sicherungsauftrag einerseits und Behandlungsanspruch andererseits, der Wahrnehmung des Abhängigen einerseits als kriminell und andererseits als krank ergeben sich strukturelle Prob- 
leme einer angemessenen medizinischen und psychosozialen Versorgung. Die in Deutschland, aber auch in den meisten europäischen Ländern vorhandene Eindimensionalität der Ausrichtung der Hilfen auf abstinenzorientierte Angebote blendet die Versorgungsnotwendigkeiten von Gefangenen mit fortgesetztem Drogenkonsum aus. Suchtbegleitung - als einer offensiv auf Schadensbegrenzung abzielenden Begleitung des Drogenabhängigen - ist innerhalb des Vollzuges so gut wie nicht entwickelt worden seit Beginn der durch das Auftreten von HIV und AIDS gekennzeichneten Phase vor 10-15 Jahren. Nur vereinzelte Projekte zielen mit Präventionsmaßnahmen und Hilfeangeboten auf Schadensbegrenzung angesichts der Verbreitung von hochriskantem Drogenkonsum und drogenbedingten Erkrankungen.

Eine offene, offensive Auseinandersetzung mit dem Problem von Infektionskrankheiten im Gefängnis unterbleibt in vielen Anstalten oder zielt lediglich auf das therapeutische Umgehen im Rahmen eines Abstinenzziels. Die gesundheitlichen und sozialen Risiken der akut intravenös Drogen gebrauchenden Gefangenen werden dabei allzu oft ausgeblendet. Ein wesentlicher Grund dafür liegt in dem vermeintlichen Widerspruch von aktivem Umgehen mit der Tatsache des Drogengebrauchs im Gefängnis und dem Vollzugsziel der Vorbereitung auf ein straffreies Leben [1].

\section{Prävalenz des Drogenkonsums in Haftanstalten}

In den letzten 25 Jahren hat sich die Insassenstruktur der etwa 220 Gefängnisse in Deutschland massiv verändert: Neben einem deutlich gestiegenen Ausländeranteil (von 9\% 1977 auf etwa 28\% im Jahre 2001) hat sich vor allem die Zahl der DrogenkonsumentInnen in Haft deutlich erhöht. Nimmt man nur die Zahl der wegen Betäubungsmitteldelikten Verurteilten, so stieg ihr Anteil von etwa $0,2 \%$ im Jahre 1970 auf etwa $14 \%$ der Gefangenenpopulation im Jahre 1999 [2, 3]. Diese Zahlen sind jedoch ergänzungsbedürftig: Viele Drogenabhängige werden aufgrund von Delikten zur Beschaffung von Drogen verurteilt oder ihr Drogenkonsum bleibt ganz und gar unerkannt. Schätzungen mehrerer Experten gehen davon aus, dass etwa ein Fünftel der Gefangenenpopulation (etwa 75300 am 30.9.2000) ehemalige oder Noch-Gebraucher von Drogen ist. Dass diese Schätzungen eher konservativ sind, zeigen in einzelnen Bundesländern durchgeführte Untersuchungen, wonach etwa jeder zweite Gefangene als „drogengefährdet" [4] und jeder Dritte als „therapiebedürftig“ [5] angesehen werden muss.

In Frauen- und Jugendhaftanstalten ist der Anteil noch höher (z.T. über 50\%; [6, 7]), und schließlich gelten diese Zahlen nur für die alten Bundesländer. In den neuen Bundesländern dominieren in etwa gleichem Maße alkholbedingte Straftaten unter den Gefangenen [2]. Küfner [8, 9] geht insgesamt davon aus, dass der Anteil Alkoholabhängiger im Gefängnis ähnlich hoch ist wie der der Abhängigen von illegalen Drogen. Für die fünf ostdeutschen Bundesländer wird der Anteil der Gefangenen mit Alkoholproblemen so hoch eingeschätzt wie im Westen das Problem mit KonsumentInnen illegaler Drogen [2].

Kreuzer [3] weist darauf hin, dass sich damit ein Zehntel der allgemein angenommenen Zahl von 15000 Drogenabhängigen in Haft befindet. Angesichts der ca. 5000 Therapieplätze befinden sich also mindestens dreimal so viele Drogenkonsumenten im Gefängnis als in therapeutischen Einrichtungen. Mehrere Studien für Deutschland $[10,11]$ bestätigen übereinstimmend, dass die von ihnen befragten DrogengebraucherInnen zu etwa zwei Dritteln Hafterfahrungen hatten - durchschnittlich mehr als 12 Monate (vgl. hier auch S.1 Suchttherapie Supplement Hepatitis). Empirische Befunde zeigen weiterhin auf, dass DrogengebraucherInnen längere Haft- als Therapiezeiten aufweisen [7, 12]. Das Gefängnis wird also zur dominanten Lebenswelt in der Biografie vieler DrogenkonsumentInnen. Diese Entwicklungen der Zunahme der Zahl der DrogenkonsumentInnen unter der Gefangenenpopulation sind europaweit zu beobachten - obwohl sich erhebliche Definitionsunterschiede von DrogenkonsumentInnen ergeben [13]. Darüber hinaus muss ein ebenfalls nicht genau festzustellender Anteil von DrogengebraucherInnen erwähnt werden, der erst in der Haft mit dem Drogenkonsum beginnt: Untersuchungen in europäischen Gefängnissen zeigen einen Anteil zwischen 5 und 25\% [14-17]. Nimmt man also $20 \%$ der Gefangenen mit einer Drogenproblematik in Haft an, so kann man von etwa 15000 inhaftierten Drogenabhängigen in Deutschlands Strafhaftanstalten ausgehen, die trotz scharfer Kontrollen entweder gegenwärtig noch (mit unterschiedlichen Konsummustern und -frequenzen) oder bis vor kurzem KonsumentIn und/oder HändlerIn illegaler und hier vor allem intravenös applizierter Drogen waren. In Europa geht man davon aus, dass ein Drittel bis zur Hälfte der 350000 InsassInnen europäischer Gefängnisse Erfahrungen mit problematischem Drogengebrauch besitzt. Berücksichtigt man die durchschnittlichen Durchlaufzahlen, dann ergibt sich eine Zahl von etwa 600000 Menschen, die mit dem System Strafvollzug jährlich in Kontakt kommen [18-21].

\section{Gesundheitliche Risilken füir drogenabhängige Inhaftierte}

Zum Zeitpunkt der Inhaftierung blicken betroffene Drogenabhängige in der Regel auf eine lange Erfahrungszeit von Drogenkonsum mit all seinen sozialen und gesundheitlichen Begleiterscheinungen zurück: gesellschaftliches Randgruppendasein, Verschuldung, Verarmung, Obdachlosigkeit, Prostitution, Gewalt, allgemeine gesundheitliche Verelendung, HIV- und/oder Hepatitisinfektion. Ist die Institution Gefängnis mit gesundheitlich und sozial belasteten Gefangenen, Überbelegung und schlechter finanzieller Ausstattung ein setting mit höherem Gesundheitsrisiko als die Lebensumstände außerhalb? Die Frage ist nicht eindeutig zu beantworten. Einerseits gelingt es einem Teil der drogenabhängigen Gefangenen, die Haftzeit für die körperliche Regeneration zu nutzen. Hier stellen sich dramatische körperliche Genesungsprozesse in relativ kurzer Zeit durch geordneten Tag-Nacht-Rhythmus, regelmäßige Ernährung, Sportangebote etc. schnell ein. Dies sind die für alle Bediensteten und Mit-Gefangenen sichtbaren Prozesse, die oft zum Umkehrschluss führen, dass das Gefängnis den InsassInnen gut tut. Andererseits stellt das Gefängnis für diejenigen, denen es nicht gelingt, abstinent zu leben, eine sehr viel höhere gesundheitliche Belastung dar. Koch/Ehrenberg [10], Weilandt [22], Weild u.a. [23] fanden übereinstimmend heraus, dass etwa die Hälfte der von ihnen Befragten ihren Drogenkonsum in Haft fortsetzt. Die Haftbedingungen bilden dann weitere soziale und gesundheitliche Belastungsfaktoren. Hier ist vor allem der ständige Wechsel von Entzug und Konsum zu nennen, oftmals überbrückt mit Ben- 
zodiazepinen oder „Aufgesetztem“ (selbst hergestellter Alkohol). Für die meisten Gefangenen gilt jedoch: Vermehrte Haftaufenthalte steigern die individuellen Problemlagen durch:

- die Fortführung des gesellschaftlichen Ausgrenzungsprozesses,

- die Festigung eines Selbstbildes als Außenseiter/in,

- die Verunselbstständigung durch hohe Passivität und das anstaltliche „Antragswesen“,

- die hohen Gesundheitsrisiken beim Drogengebrauch selbst und den konsumbedingten Folgen (Überdosierungen, Abszesse, Mischintoxikationen, Infektionsgefahren viraler Hepatitiden und HIV/AIDS).

Besonders anschaulich wird die Spirale der Verelendung von Wedershoven [24] beschrieben, die in ihrer katamnestischen Untersuchung HIV-infizierter Gefangener in einer nordrheinwestfälischen HIV-Ambulanz eines Justizvollzugskrankenhauses aufzeigt, dass psychische, soziale und gesundheitliche Krisen in den Lebensverläufen der untersuchten Gefangenen den Charakter einer Eskalation annehmen, ein Prozess, in dem die HIV-Infektion allein für die praktische Lebensbewältigung der meisten Patienten sogar zunächst nachrangig ist.

Exemplarisch für die gesundheitlichen Belastungen seien drei Beispiele von Risiken herausgehoben [25]:

1. Drogenkonsum: I.v.-Drogenkonsum ist bei den Drogenabhängigen zwar weniger verbreitet als außerhalb, aber die Gefangenen, die ihren Konsum im Gefängnis fortsetzen, tun dies unter hoch riskanten Bedingungen und in der Regel mit einem Gemeinsamgebrauch von Spritzen, Nadeln und Spritzutensilien.

2. Sexuelle Beziehungen: Zwar gehen weniger Gefangene sexuelle Beziehungen ein als außerhalb, aber obwohl die Kondomnutzung außerhalb schon gering ist, ist sie im Vollzug praktisch nicht existent.

3. Tätowieren/Piercen: Diese Praxis ist im Vollzug weit verbreitet und wird in der Regel unter sehr unhygienischen Umständen durchgeführt.

Ad 1. Gemeinsambenutzung gebrauchter Spritzen und das Teilen von Drogen und Konsumutensilien

Über die Gemeinsambenutzung von unsterilem Spritzbesteck und dem ebenfalls riskanten Teil der Drogen und der Konsumutensilien gab es zunächst nur vereinzelte anekdotische Berichte. Darin kommt immer wieder zum Ausdruck, dass es sehr grobe und unzuverlässige Vorsichtsmaßregeln gibt („Positive spritzen als letzte“), Spritzen unter klarem oder warmem Wasser ausgespült oder ausgekocht werden (nicht in jeder Anstalt sind Tauchsieder zulässig) oder auf diese Vorsichtsmaßnahmen in der Dynamik der Situation völlig verzichtet und die „Stationspumpe" schlechten Gewissens benutzt wird. Wedershoven [24] bestätigt, dass unsterile Spritzutensilien die Hauptinfektionsquelle der von ihr untersuchten Gefangenen darstellten. Als Faktoren, die einem risikoarmen Drogenkonsum entgegenstehen, benennt sie: Probleme in der primären Sozialisation, Polytoxikomanie, Alltagsstressoren, lange Drogenkarriere und Haftaufenthalte (S. 92).

Knapp [26] fand, dass bei den von ihm befragten 44 HIV-AK-positiven Strafgefangenen etwas mehr als die Hälfte angab, dass bis zu neun Personen eine umlaufende Spritze gebrauchten. Etwas weniger als die Hälfte gab an, dass in der Regel mindestens zehn Gefangene je Spritze beteiligt seien. Davon gaben 32\% an, dass mindestens fünf Personen beteiligt seien.

Bereits von Studien außerhalb des Gefängnisses wissen wir, dass die Drogeninjektion ein besonders kommunikativer und kooperativer Akt ist [27]. Absprachen bez. vorhandener HIV-Infektion müssen als unzuverlässig bezeichnet werden; sie vermitteln eine Scheinsicherheit; Reinigungen können zudem nicht mit der erforderlichen Sorgfalt vorgenommen werden [28]. Insbesondere vor dem Bekanntwerden von HIV/AIDS existierte die Form des Drogenkonsums in Haft, die als klassisches needle sharing zu bezeichnen ist. Bei der thermischen oder chemischen Desinfektion ist ebenfalls kein sicherer Umgang zu erwarten, denn es bestehen in den Strafvollzugsanstalten und bei den Gefangenen in der Regel keine Gebrauchsanweisungen für eine effektive chemische (mit bleach, d.h. Haushaltsbleicher: Welche Mittel sind wie eingesetzt, geeignet, Viren abzutöten?) oder thermische Desinfektion (Auskochen: wie lange mit welchen Spritzen machbar?).

Mehrere Untersuchungen bestätigen Ansteckungen von Hepatitis und HIV in den Gefängnissen [29, 30].

\section{Ad 2. Sexualität in Haft}

Ebenso wenig wie der Strafvollzug eine drogenfreie Zone ist, ist er ein sexualfreier Lebensraum. Sexuelle, partnerInnenorientierte Bedürfnisse sind nach wie vor bei den Gefangenen vorhanden, können allerdings in der Regel nicht mehr ausgelebt werden. Der Strafvollzug hat auch in diesem Bereich von Lebensäußerung schädliche Wirkungen. Zwar existieren vereinzelt Modelle in Strafanstalten, die im Rahmen von Langzeitbesuchen auf eine Ermöglichung auch sexueller Kontakte unter (Ehe-)PartnerInnen zielen (z.B. JVA Werl, JVA für Frauen Vechta), und lockerungsberechtigte Häftlinge können im Urlaub sexuelle Kontakte haben. Doch dies sind vereinzelte und isolierte Möglichkeiten, partnerInnenorientierte Sexualität zu leben. In der Haft ist Sexualität einem Tabu vergleichbar [31]: Symbolisierungen sind allgegenwärtig, eine sachliche Verbalisierung findet kaum statt. In Ermangelung heterosexueller Kontakte im Strafvollzug sind nur gleichgeschlechtliche soziale Sexualkontakte lebbar. Diese sind z.T. noch stärker als das Phänomen Drogenkonsum tabuisiert und verdrängt. „Unterschwellig scheint das ,Verbot” der Ausübung von Sexualität nach wie vor als Teil der Strafe angesehen zu werden" [32]. Die Gefangenen werden mit den gleichen Mechanismen konfrontiert wie die Gesamtgesellschaft: Einer Sexualisierung vor allem des weiblichen Körpers in den Medien wird hauptsächlich mit einer ,offensiven Strategie‘ der Thematisierung von Sexualität begegnet. Durch eine Verobjektivierung des weiblichen, z.T. auch des männlichen Körpers in Form von Postern an den Zellenwänden und einer starken Präsenz sexualitätsbezogener Gesprächsinhalte drückt sich der entfremdete Umgang mit den eigenen sexuellen Bedürfnissen aus. Der Objektstatus des sexualisierten Körpers reduziert wiederum die eigene Empfindungsspanne und verleugnet die mit partnerInbezogener Sexualität assoziierten Bedürfnisse nach Nähe, Gemeinsamkeit, Entspannung, Befriedigung. Das Dilemma, vor allem im Männervollzug, besteht in der Allgegenwärtigkeit von Sexualität im Alltag und der stark eingeschränkten Befriedigung und letztlich erzwungenen Milieuanpassung sexueller Bedürfnisse. Da- 
raus erwachsen Spannungen, Frustrationen, Aggressionen, sexualisierte Gewaltphantasien. Vorhandene Probleme mit diesem Dilemma können nicht besprochen werden, weil weder informelle noch offizielle Foren im Vollzug zur Verfügung stehen.

Weil Sexualität individuell abgespalten werden muss und die Thematik Sexualität im Vollzug offiziell ausgeblendet wird, finden alle Formen gelebter sozialer Sexualität verdeckt statt. Es gibt eine Realität von gleichgeschlechtlichen Sexualkontakten, aber auch Homosexualität, die kaum thematisierbar ist. Der Geheimhaltungsdruck verstärkt sich vor allem dann, wenn die in Haft gelebten homosexuellen Kontakte nicht dem eigenen sexuellen Selbstverständnis „heterosexuell“ entsprechen, was bei vielen Inhaftierten der Fall sein wird, die in der Inhaftierungszeit in Ermangelung heterosexueller Möglichkeiten homosexuelle Kontakte als „Notlösung“ praktizieren. Soziokulturelle Barrieren einer Zwangsheterosexualität als gesellschaftliche Normalität und Homophobie wirken in dieser doppelten Realität der sexuellen Identität, die sich in der praktizierten Sexualität im Vollzug nicht wiederfindet. Wenn diese „Notlösungen“ über lange Zeit zur Gewohnheit sexueller Aktivität werden, repräsentieren sie Normalität unter den Inhaftierten, ohne offizielle Anerkenntnis und Verantwortungsübernahme. So entwickelt sich Homosexualität als Dunkelfeld, wo Prostitution z.B. zur Drogenbeschaffung oder Vergewaltigungen stattfinden, ohne offen als Realität anerkannt zu werden.

\section{Ad 3. Tätowieren}

Die Verwendung von nichtsterilen Nadeln beim Piercen oder Tätowieren kann ebenso wie die Verwendung unsteriler Nadeln beim i.v.-Drogenkonsum zur Übertragung von Infektionskrankheiten führen. Welches Ausmaß Tätowieren als infektionsrelevantes Verhalten unter Strafgefangenen immer noch besitzt, ist nicht wissenschaftlich erhoben worden. Auch andere Untersuchungen [26] weisen zwar darauf hin, dass diese Praktiken der Tätowierung zwar nicht irrelevant sind für den Strafvollzug, dass aber kein Wissen über die Verbreitung der Tätowierung unter insbesondere unsterilen Bedingungen bei den Gefangenen oder den Bediensteten herrscht. Eine Bereitstellung von Desinfektionsmitteln zum Sterilisieren oder Möglichkeiten zum Auskochen der Nadeln würde Ansteckungsgefahren drastisch reduzieren. Da Tätowieren in der Regel eher akzeptiert wird als das Injizieren von Drogen (in Europa ist 30\% des Gefängnispersonals selbst tätowiert), lässt sich diese Vorbeugungsmaßnahme leichter einführen als die Ausgabe von Bleichmitteln zum Sterilisieren von Spritzen. Die Bereitstellung von Bleichmitteln zum Reinigen der Tätowierinstrumente könnte sogar den Weg ebnen für die Ausgabe von Bleichmitteln für Drogenabhängige.

Ein Gesamtbild über die gesundheitliche Lage Drogenabhängiger in deutschen Haftanstalten existiert nicht, wie generell ein Mangel an aggregierten Gesundheitsdaten auch innerhalb der Länder zu verzeichnen ist. Sollen gesundheitliche Entwicklungen erforscht werden, so ist die Durchsicht entsprechender Gesundheitsakten vonnöten.

Ergebnisse einer Vergleichsuntersuchung der Gesundheit der Gefangenenpopulation mit der allgemeinen Bevölkerung in der Schweiz legen allerdings nahe, dass auch für Deutschland - ausgehend von der Tatsache einer hohen Zahl gesundheitsbelasteter
Gefangener im riskanten setting Strafvollzug - der allgemeine Gesundheitszustand der Gefangenen, verglichen mit der Allgemeinbevölkerung, als deutlich schlechter zu bewerten ist [33].

Was wissen wir über die Verbreitung von HIV/AIDS und viralen Hepatitiden (v.a. B+C) im Gefängnis?

\section{Hepatitiden}

Im „risk-environment“-Justizvollzug scheinen Hepatitisinfektionen sehr viel stärker verbreitet zu sein als außerhalb. Mit der Fokussierung und Reduktion der gesamten Aufmerksamkeit auf das HI-Virus in den Gefängnissen ist das Infektionsrisiko Hepatitis in den letzten Jahren in den Anstalten massiv unterschätzt worden. Doch gerade hier bestehen erhebliche - vielfach auch von den Landesjustizbehörden völlig vernachlässigte - Risiken einer schon als „gefängnistypisch“ zu bezeichnenden Verbreitung vor allem bei i.v.-Drogenkonsumenten [34].

Mittlerweile ist jedoch auch der Strafvollzug für diese Infektionskrankheiten sensibilisiert. Dazu haben verschiedene Forschungs- und Dokumentationsarbeiten über Prävalenzen und Inzidenzen beigetragen. Gaube et al. [34] fanden in der Justizvollzugsanstalt (JVA) Wolfenbüttel eine 100-200fach erhöhte Häufigkeit der Verbreitung der drei Hepatitiden A, B und C im Vergleich zur Normalbevölkerung. Keppler et al. [29] fanden bei einer Untersuchung in der niedersächsischen Justizvollzugsanstalt für Frauen in Vechta bei drogenkonsumierenden Frauen Infektionsraten von $78 \%$ für Hepatitis B und 74,8\% für Hepatitis C. Darüber hinaus wurden während der Haftzeit in nicht unerheblichem Umfang Neuinfektionen festgestellt: Von den 41 serokonvertierten (= neuinfizierten) Patientinnen hatten sich 20 $(48,8 \%)$ ihre Hepatitisinfektion während der Haft erworben (s.u.). Auch in anderen europäischen Strafvollzugseinrichtungen wird eine ähnlich hohe Verbreitung von Hepatitis B und $C$ berichtet [35]. Stark et al. [36] weisen in ihrer Untersuchung über IDU in Berlin ( $\mathrm{n}=324$ ) darauf hin, dass ein unabhängiger Risikofaktor für die Infektion mit HIV, HBV und HCV „needle sharing“ im Gefängnis war. Holsen et al. [37] kommen in ihrer Untersuchung über die Prävalenz von HCV in einem Gefängnis in Norwegen zu den Ergebnissen, dass 46\% der untersuchten Gesamtgruppe, davon jedoch $73 \%$ in der Untergruppe der IDU, HCV positiv waren, was einer ca. 400fach höheren Verbreitung als bei Blutspendern (als Vergleichskollektiv aus der „Allgemeinbevölkerung“) entsprach! Nur etwa die Hälfte (47\%) der HCV-positiven Gefangenen war sich über ihre Hepatitiserkrankung im Klaren.

Gbordzoe [38] fand bei einer Untersuchung in der JVA Heilbronn unter allen Gefangenen eine Verbreitung von Hepatitis B von $3,1 \%$ und von Hepatitis C von $17 \%$. In der Gruppe der IDU konnte jedoch eine Prävalenz der Hepatitis C von $77 \%$ ermittelt werden. Bredeek et al. [39] fanden bei 621 HIV-Positiven, die zwischen 1983 und 1995 im Berliner Justizvollzug zusätzlich auf Hepatitis untersucht wurden, bei 95,8\% auch serologische Marker für eine durchgemachte oder aktive Hepatitis B, von den seit Verfügbarkeit des Hepatitis-C-Tests 1990 auch auf HCV-Antikörper untersuchten 271 Insassen erwiesen sich $97 \%$ als positiv! 
Die medizinische Begleitforschung des „Pilotprojektes zur Infektionsprophylaxe für Drogenabhängige in der Anstalt des offenen Vollzugs in Vierland (Anstalt XII)“ erbrachte Gesamtprävalenzen für Hepatitis $B$ und $C$ bei allen untersuchten Gefangenen von etwa $50 \%$ zu zwei verschiedenen Zeitpunkten [35]. Bei genauerer Betrachtung von Untergruppen wurde festgestellt, dass die Gruppe der Heroin-/Kokaingebraucher in Haft wesentlich höhere Prävalenzen zu zwei unterschiedlichen Zeitpunkten aufwiesen (Hepatitis B 64\% bzw. 80\%; Hepatitis C 89\% bzw. 81\%).

Eine schriftliche Befragung der Landesjustizbehörden [22] verdeutlichte, dass Eingangsuntersuchungen auf Hepatitiden nicht routinemäßig, sondern nur bei medizinischer Indikation durchgeführt werden. Positive Hepatitis-Befunde werden bisher von keiner der Landesbehörden erfasst. Eine Ausnahme bildet die Praxis in Baden-Württemberg: Auf Veranlassung des Justizministeriums Baden-Württemberg gehört seit 1995 die Hepatitis-C-Serologie grundsätzlich zur Eingangsuntersuchung der Gefangenen. Nach den vorliegenden Erkenntnissen weisen etwa $17 \%$ der Gefangenen einen positiven Hepatitis-C-Befund auf der Anteil Drogenabhängiger darunter beträgt etwa $70 \%$, wobei die Hälfte dieser Gefangenen als infektiös eingestuft wird. Zwei Strafgefangene haben sich wahrscheinlich während der Inhaftierung mit HCV infiziert, ein weiterer wohl mit HIV [40]. Demnach erfordern infektionsprophylaktische Bemühungen insbesondere im Justizvollzug eine über die Beachtung und Eindämmung einer HIV-Verbreitung hinausgehende Realisierung des erheblichen Infektionsrisikos Hepatitis unter IDU.

\section{HIV/AIDS}

Die Prävalenzen der HIV-infizierten Inhaftierten wird in Deutschland von offizieller Stelle [41] bei den Männern von $0,12 \%$ bis $2,8 \%$ und bei den Frauen von $0,48 \%$ bis $8 \%$ angegeben (Anfang 1994 waren den Justizbehörden bekannt: 406 Männer und 55 Frauen HIV-positiv, 42 Männer und 15 Frauen an AIDS erkrankt). Dies wird durch mehrere Untersuchungen zur Prävalenz von HIV unter Gefangenen bestätigt [29, 38]. Die zahlenmäßig größte Studie zur HIV-Prävalenz durchgeführt im Hamburger Justizvollzug in den Jahren 1993-1995 ( $\mathrm{n}=19482)$, zeigt eine konstante Verbreitung zwischen 1,1 und 1,6\%, allerdings einen Anstieg bei den i.v. applizierenden Gefangenen [42]. Dies zeigt, dass HIV/AIDS im Gefängnis im Wesentlichen unter IDU verbreitet ist. Nimmt man für Deutschland schließlich eine HIV-Prävalenz unter den Gefangenen von ca. $1 \%$ an, bedeutet dies eine 25-mal höhere HIV-Verbreitung in der Gefangenenpopulation, als für die Durchschnittsbevölkerung angenommen wird.

Mehrere Studien außerhalb des Strafvollzugs belegen eine enge Korrelation zwischen einem ehemaligen Aufenthalt im Strafvollzug und der Verbreitung o.g. Infektionskrankheiten [43]: Hafterfahrungen bei IDU stellen sich als signifikanter Risikofaktor für eine HIV-Infektion dar [ausführlich: 44-46]. Zu bedenken ist, dass die HIV-Testraten bei Gefangenen von Bundesland zu Bundesland sehr unterschiedlich sind und in einigen Bundesländern nur in sehr viel geringerem Umfang getestet wird: Bei den Eingangsuntersuchungen mit über $90 \%$ in Hamburg und Bayern vs. ca. $10 \%$ in Bremen! Es bleibt also noch ein erhebliches Dunkelfeld von nichtgetesteten Gefangenen.
Konsequenzen fürr die Gesundheitsfürrsorge im Gefängnis

Der Strafvollzug steht insbesondere unter dem Druck der Öffentlichkeit, das Gefängnis als ,drogenfreien Raum` zu gestalten. Nur wenige Anstalten sprechen offen über vorhandene Drogenprobleme und bieten offensive Hilfen an: Dies wird oft gleichgesetzt mit einem Scheitern des Sicherheitsauftrags und einer Desillusionierung in Bezug auf Undurchlässigkeit des Gefängnissystems. Nach wie vor gibt es eine erhebliche Anzahl von Anstalten, die einen Drogengebrauch entweder nicht beobachten oder ihn aus justizpolitischen Motiven ignorieren und leugnen. Unter diesem Umstand, dass eigentlich ,nicht sein kann, was nicht sein darf', ist der Umgang mit Drogenabhängigen im Vollzug ein Balanceakt zwischen einerseits den Erwartungen an die Erfüllung seines Resozialisierungsauftrags und andererseits den Realitäten eines verbreiteten Drogenkonsums vieler Gefangener und z.T. politisch stark eingeschränkter Handlungsmöglichkeiten zur Bewältigung dieses Phänomens. Gegenüber diesen Entwicklungen existieren lediglich zwei Reaktionsmuster: Zum einen kann versucht werden, den Strafvollzug noch weiter abzuschotten. Sofern dadurch Drogenzufuhr und -konsum überhaupt reduziert werden könnten, ist dies vor dem Hintergrund eines resozialisierenden Auftrags mit dem Ziel weitgehender Außenorientierung des Strafvollzugs kritisch zu betrachten. Zudem würde diese Maßnahme zu Lasten auch nicht betroffener Gefangener gehen. Zum anderen kann man innerhalb des Spannungsfeldes von Sicherheit und Ordnung auf der einen und Hilfsangeboten auf der anderen Seite realitätsgerechte und bedürfnisgerechte Hilfen entwickeln. Diese sollten pragmatische Ziele verfolgen, machbar sein sowohl für den Vollzug als auch für die Gefangenen. Deutlich wird allerdings, dass die Arbeit mit DrogenkonsumentInnen im Justizvollzug nur unter sehr eingeschränkten räumlichen und methodischen Bedingungen stattfinden kann. Drogenszene und Hilfeangebote fallen räumlich zusammen, es gibt keine Toleranz des Konsums: Sicherheits- und Ordnungsaspekte strukturieren und dominieren klar den Anstaltsalltag und die Umgehensweise mit den drogengebrauchenden Gefangenen.

Ein weiteres Beispiel für die Schwierigkeit des Umgangs mit Drogenabhängigkeit besteht darin, dass sie in der Regel als „nicht lockerungsgeeignet" betrachtet werden. Die Praxis in einigen Bundesländern (Niedersachsen und Hamburg), besteht darin, Lockerungen nach Kriterien der Auffälligkeit zu handhaben. Offenbar existiert sowohl in dieser Frage als auch in der Frage der Eignung bzw. Ungeeignetheit von DrogenkonsumentInnen für den Offenen Vollzug ein Nord-Süd-Gefälle. Eine Allgemeine Verfügung der Hamburger Justizbehörde stellt diesbezüglich klar, „dass eine Nichteignung nur dann vorliegt, wenn die Suchtgefährdung eine Flucht- oder Missbrauchsgefahr begründet" [47]. Gerade der Eingliederungsgrundsatz ( $§ 3$ Abs. 3 StVollzG), wonach Probehandeln in der Freiheit ermöglicht werden soll, wird mit dem Hinweis auf die bestehende Suchtgefährdung verunmöglicht.

Gesundlheitsfürrsorge und handlungsleitende Zielorientierungen im Umgehen mit DrogengebraucherInnen

Die Gesundheitsfürsorge für die Gefangenen liegt in der Verantwortung der Justizvollzugsbehörden. An die Stelle von Eigenverantwortlichkeit und Selbstbestimmung tritt die Fürsorge der An- 
stalt und die Verpflichtung des Gefangenen, die notwendigen medizinischen Maßnahmen zu unterstützen. Durch den Freiheitsentzug steht der Gefangene in einem besonderen Abhängigkeitsverhältnis zum Staat, woraus sich eine besondere Verantwortung und Fürsorgepflicht des Staates gegenüber dem Gefangenen ergibt. Die Bedeutung der physischen und psychischen Gesundheit für die Gefangenen muss als die zentrale Voraussetzung für die Wiedereingliederung in das Alltags- und Erwerbsleben betrachtet werden. Bei der Normierung der Gesundheitsvorschriften sah sich der Gesetzgeber zunehmend verpflichtet, die zunächst geplante Übertragung der Leistungen der gesetzlichen Krankenversicherung zugunsten besonderer Regelungen im Vollzug einzuschränken. Das Gesetz geht davon aus, dass die medizinische Versorgung durch hauptamtliche Ärzte und qualifiziertes Krankenpflegepersonal sichergestellt wird (§158 Abs. 1). Diese Regelung soll garantieren, dass sowohl für die regelmäßig anfallenden Aufgaben als auch in dringenden Fällen ein Arzt schnell hinzugezogen werden kann. Die Praxis zeigt, dass in vielen Anstalten Vertragsärzte angestellt sind, die lediglich zu festgelegten Zeiten in den Anstalten für Gefangene erreichbar sind. Die über die VV in den einzelnen Bundesländern geltenden Vorschriften sind dort zusätzlich in einer „Dienstordnung Gesundheit" (DOG) oder in einem vergleichbaren Regelwerk zusammengestellt.

Woran sollte sich Drogenhilfe im Gefängnis orientieren? Zunächst ist der Angleichungsgrundsatz im Strafvollzugsgesetz für eine anstaltsinterne Anpassung an fachliche Standards der Drogen- und AIDS-Hilfe außerhalb von großer Bedeutung: Grundsätzlich hat sich Anstaltsmedizin an den Vorgaben der Gesetzlichen Krankenversicherung zu orientieren - Strafvollzug soll primär nur Freiheit nehmen, aber nicht durch schlechtere medizinische Versorgung zusätzlich bestrafen. Dieses Äquivalenzprinzip soll die Vergleichbarkeit intra- und extramuraler medizinischer Versorgung gewährleisten. Die Grundlagen für dieses Äquivalenzprinzip bestehen in dem gesetzlichen Auftrag zur Angleichung der Lebensverhältnisse und Gegenwirkung schädlicher Wirkungen des Freiheitsentzuges ( $\$ 3$ Abs. 1 und 2). Diese Grundsätze verlangen eine Orientierung an den außerhalb erprobten und bewährten Standards und Leitlinien als Richtschnur intramuraler Versorgungs-, Beratungs- und Behandlungsangebote. Dies betrifft auch die psychosozialen Hilfen innerhalb des Vollzuges: „Es ist alles zu versuchen, um Gefangene nicht kranker aus dem Vollzug zu entlassen, als sie hereingekommen sind: Das ist das Minimum an vorbeugender Gesundheitsfürsorge, das im Vollzug zu leisten ist“ " 48 ]. Hierzu sind realistische Einschätzungen und Untersuchungen über die den Gefangenen im Vollzug drohenden gesundheitlichen Risiken und dementsprechende anstaltliche Maßnahmen und Mittel der Risikovorsorge erforderlich.

Das Äquivalenzprinzip ist über ethische und fachliche, international (siehe AK) fixierte Grundsätze hinaus aber auch von enorm praktischer Bedeutung: Nur durch eine Angleichung der Standards in der Praxis kann eine wirksame Vernetzung der Hilfen vor und nach der Entlassung in die Kommune gewährleistet werden. Das heißt, dass die Maßnahmen zur allgemeinen Gesundheitsfürsorge im Vollzug in enger Zusammenarbeit zwischen nationalen Gesundheitsbehörden, Gefängnisverwaltungen und Zielgruppenvertretern, einschließlich regierungsunabhängiger Organisationen ausgearbeitet werden sollten. Und sie sollten in ein breites Programm der Gesundheitsförderung eingebettet sein. Schließlich sollten die Bedürfnisse von Gefangenen und anderen Personen in der Institution Gefängnis (der Bediensteten) Berücksichtigung finden.

Eine wesentliche Einschränkung dieses Äquivalenzprinzips besteht in der Aufhebung des Prinzips der freien Arztwahl, da Kostenträger nicht die gesetzliche Versicherung, sondern die Vollzugsbehörde ist. Gefangene in offenen Anstalten, die als pflichtversicherte Arbeitnehmer beschäftigt sind, haben dagegen die Berechtigung, einen Arzt ihrer Wahl aufzusuchen (§62a). Alle anderen Gefangenen sind primär auf den Anstaltsarzt angewiesen. Sie haben nur eingeschränkt die Möglichkeit, wie ein Patient außerhalb des Vollzugs auf eigenen Wunsch zu einem anderen Arzt überwiesen zu werden, um Zweifel an der Diagnose zu klären und über die Behandlung mitzuentscheiden. Zwar kann die Anstalt weitere Ärzte hinzuziehen oder dem Gefangenen gestatten, auf eigene Kosten einen freien Arzt aufzusuchen, wenn der Anstaltsarzt dies befürwortet oder nicht erreichbar ist; die Entscheidung liegt jedoch allein bei der Behörde. Die Einbeziehung aller Gefangener in die gesetzliche Krankenversicherung ist bis zum Erlass eines besonderen Bundesgesetzes aufgeschoben (§198 Abs. 3). Erst hierdurch werden die Voraussetzungen geschaffen, dass auch die Gefangenen entsprechend dem Angleichungsgrundsatz die für die Allgemeinheit zugänglichen medizinischen Dienste in Anspruch nehmen können, dass eine wesentliche Ursache von Angst und Misstrauen wegfiele.

Der Nachteil dieses Konzepts der Gesundheitsfürsorge liegt einmal darin, dass es eine erhebliche Belastung der Arzt-Patient-Beziehung mit sich bringt, wenn der Arzt 1 . in die Hierarchie des Anstaltspersonals integriert ist, 2 . Kontrollaufgaben für die Institution wahrnimmt (z.B. Urinkontrollen) und 3. die Kontaktaufnahme des Patienten nicht freiwillig erfolgt. Der Anstaltsarzt wird zum „Zwangsansprechpartner“ innerhalb des Spannungsverhältnisses von kontrollorientierten Vorgaben und Zwängen des Vollzugs und einer patientenorientierten Behandlung. Dieses strukturelle Problem fördert gegenseitiges Misstrauen und belastet die gesundheitliche Versorgungsqualität für die Gefangenen. Unter normalen Bedingungen ist der Vertrauensarzt der Krankenkasse, des Arbeitsamtes, des Gesundheitsamtes oder der Betriebsarzt nicht gleichzeitig behandelnder Arzt. Zum anderen dürften die in diesem Titel geregelten Leistungen nicht allein durch hauptamtliche Ärzte zu erbringen sein, so dass das Hinzuziehen von Fachärzten ( $\S 58$ VV Nr. 2 Abs. 2) der Regelfall sein muss. Die Vollzugsbehörde ist nicht in der Lage, die ganze Palette von Spezialisten anzustellen und ihnen die notwendige Praxiseinrichtung zur Verfügung zu stellen.

Die Ziele und die Praxis der AIDS- und Drogenhilfe im Vollzug sollen und können sich also nicht grundsätzlich von denen in Freiheit unterscheiden. Die Angebote, die sich in Freiheit bewährt haben, müssen auch in Haft als Orientierungsgrundlage für die Ausgestaltung der Suchtkrankenhilfe dienen. Allerdings ist die Phase der Haft ein denkbar ungünstiger Zeitpunkt, um einschneidende und nachhaltige Verhaltensänderungen erwarten zu können. Gleichwohl kann der Gefängnisaufenthalt ein wichtiger Baustein sein, Abstinenz und körperliche Verbesserung wieder oder überhaupt wieder zu erleben. Die Zwangsbedingungen können insofern motivierend wirken - sie können aber auch - insbesondere bei mangelhafter Personal- und Sach- 
mittelausstattung - demotivierend und konsumverfestigend bis -steigernd wirken (zu den therapiewidrigen Rahmenbedingungen der Haft: [3]).

Drogenarbeit im Vollzug sollte lebensweltnahe Veränderungsangebote entwickeln, ohne ausschließlich und oft unrealistisch Forderungen nach abstinenter Lebensweise für jeden Gefangenen zu stellen: Eigenmotivation und Selbstverantwortung sollten geweckt, die Gefangenen befähigt werden, die haftbedingten Einschränkungen zu verarbeiten und ihre Geschicke wieder selbst in die Hand zu nehmen. Von einem „empowerment“-Gedanken, der beinhaltet, den Rahmen für individuelle Förderungen bereitzustellen (siehe Keppler in diesem Heft), ist die totale Institution Gefängnis jedoch noch weit entfernt. Eigenständigkeit, Initiative und Aktivität werden immer wieder gebremst. Gleichwohl kann die Betroffenenkompetenz gewürdigt und genutzt und in Peer-support-Ansätzen in die anstaltliche Drogenhilfe eingebunden werden [49].

Neben der klassischen Ausrichtung sollten deshalb auch pragmatische, erreichbare Ziele verfolgt werden, die sich zunächst auf Strategien der Schadensminimierung beziehen und damit eine hohe Priorität genießen.

\section{Drogenhilfen im Gefängnis}

Während sich draußen eine Vielzahl verschiedenschwelliger Hilfen mit unterschiedlichen Zielsetzungen in den letzten 10-15 Jahren entwickelt haben (suchtbegleitender Ansatz, ambulante Therapie, Substitutionsbehandlung, Originalstoffvergabe etc.), so ist das Behandlungsangebot für Drogenabhängige im Vollzug überwiegend immer noch reduziert auf abstinenzorientierte Hilfen [50]. Diese Zielorientierung ist identisch mit dem Vollzugsziel (legale und illegale Drogen sind im Vollzug verboten, und das Ziel des Gefängnisses ist es, auf ein Leben ohne Straftaten vorzubereiten - das schließt illegalen Drogenumgang per se aus) und vermittelt eine Eindeutigkeit, die intra- und extramural eine große sozialpsychologische und politische Bedeutung erhält. Drogenkonsum im Gefängnis mit all seinen Risiken kann so in einer Ausblendung von Ambivalenz und Reduktion von Komplexität erfolgreich ausgeklammert werden.

Im Folgenden werden die Behandlungsangebote im Vollzug aufgeführt und problematisiert:

Entzugsbehandlung: Entgegen den entwickelten therapeutischen Standards des Entzugs außerhalb [51] findet diese Differenzierung der Angebote in den Gefängnissen oft keine Entsprechung. In vielen Haftanstalten wird der Entzug ohne medikamentöse Hilfe durchgeführt, d.h., Gefangene werden in der kritischen Phase mit ihren Entzugssymptomen oft allein gelassen und nicht ausreichend oder nicht rechtzeitig behandelt, oder die Probleme werden überhaupt nicht erkannt, weil die Gefangenen aus Angst vor Stigmatisierung und vollzuglichen Restriktionen ihre Opiatabhängigkeit verheimlichen.

„Drogenfreie Stationen/Zonen“, Abstinenzerprobungsabteilungen sind Bezeichnungen für anstaltsinterne Bemühungen besonders motivierter Gefangener, eigene vollzugsinterne Behandlungsangebote zu unterbreiten oder/und sie zügig in eine Therapie überzuleiten. Die drogenfreien Zonen sind räumlich abgetrennte Einheiten, um eine Distanz zu ggf. konsumierenden Insassen zu schaffen. Die Gefangenen verpflichten sich (teils per Vertrag) zur Abstinenz, ihr Abstinenzstatus wird dann per (zufälligen) Urinkontrollen überprüft. Dieses Angebot wird (ehemaligen) DrogenkonsumentInnen nur in einigen Anstalten Deutschlands unterbreitet. In NRW beispielsweise hat man damit begonnen, in den 37 Justizvollzugsanstalten besondere Abteilungen für Präventions- und Motivationsarbeit einzurichten (bisher in 13 umgesetzt; vgl. [52]). Diese drogenfreien Abteilungen (s. o.) zeichnen sich durch ein interdisziplinär arbeitendes Team, einen besseren Personalschlüssel, Freiwilligkeit der Aufnahme und Drogenscreenings zur Unterstützung der Abstinenz aus. Für die Mitarbeiter in diesen Abteilungen gilt die Schweigepflicht - gleichwohl wird die Einhaltung oftmals von Gefangenen skeptisch betrachtet. In der Regel besteht keine wissenschaftliche Begleitung (Ausnahme: Evaluation der abstinenzorientierten Behandlungsstrategien in der JVA Vierlande in Hamburg: Heinemann/ Bohlen/Püschel in dieser Ausgabe). Es zeigt sich, dass Teilnehmer, die das Programm regulär abschlossen und eine Anschlusstherapie durchlaufen, eine hohe Legalbewährungszeit aufweisen.

Therapeutische Gemeinschaften mit eigenem Lebensgemeinschafts- und Arbeitsanspruch sind innerhalb des Strafvollzugs in deutschen Gefängnissen (im Gegensatz zu England und Wales) nicht bekannt. Gleichwohl bestehen in vielen Gefängnissen Selbsthilfegruppen für Alkoholiker (AA, Blaukreuz, Guttempler etc.) und auch für KonsumentInnen illegaler Drogen (NAs, JES, AIDS-Hilfen).

Eine interne Suchtberatung findet in mehreren Bundesländern (z.B. NRW, Niedersachsen) statt, z.T. mit Personal aus dem allgemeinen Vollzugsdienst, z.T. und in geringerem Umfang mit ausgebildeten Fachkräften. Die Aufgabe besteht darin, Motivationsarbeit für eine Therapieaufnahme zu leisten, Unterstützung in Krisensituationen, Einzelberatungen, Gruppenangebote zu leisten. Die JVA für Frauen bietet ganz unterschiedliche Selbsthilfe- und Gesprächsgruppen (z.T. unter Beteiligung und Anleitung der lokalen AIDS-Hilfe oder Drogenselbsthilfe JES) für gesundheitlich und sozial belastete Frauen an. Auch Safer-use- und Safer-sex-Trainings werden angeboten. Die Aufgabe der internen SuchtberaterInnen ist es ferner, unter Wahrung des Datenschutzes mit allen sozialen Diensten und der medizinischen Abteilung zu kooperieren und eine enge Verbindung zu den Mitarbeiterlnnen der externen Suchtberatung herzustellen.

Externe Drogenberatung: Aus der Erkenntnis, dass Drogenprobleme von Gefangenen innerhalb des Vollzugs kaum gelöst, sondern allenfalls Hilfen zur Bewältigung während der Haftzeit gegeben werden können, haben sich in fast allen Bundesländern externe Drogenberatungen im Vollzug etabliert. Die Einbindung dieser externen Fachkräfte mit ihrer Kompetenz und ihrem Wissen über Drogenhilfeangebote in der Region oder der Kommune schafft die notwendige Voraussetzung für eine Überleitung in die Freiheit, wo die Gefangenen nach ihrer Entlassung oder aus der Haft heraus Probleme ihrer Drogenabhängigkeit besser bearbeiten können. Die Vorteile der Einbeziehung externer Drogenberatungen - unter Wahrung ihres Externenstatus - sind Freiwilligkeit ihrer Inanspruchnahme, die Anonymität, mit der diese Hilfen nicht zuletzt aufgrund des Zeugnisverweigerungsrechts 
der Drogenberatungsstellen geleistet werden können, und die Unabhängigkeit vom vollzuglichen Auftrag. Ein wesentlicher Vorteil externer Drogenberatung ist schließlich die Möglichkeit der Weiteranbindung nach Haftentlassung: Dadurch werden längerfristige Betreuungskontakte mit AnsprechpartnerInnen möglich, die Kontinuität und Verbindlichkeit gewährleisten. Dieser Dienst bildet die Brücke zwischen Gefängnis- und Gemeindediensten.

Die inhaltliche Orientierung dieser Arbeit erfolgt oftmals an den Zielsetzungen und Tätigkeitsmerkmalen der Drogenberatungsstellen außerhalb des Vollzugs. Für die spezifische Arbeit mit straffällig gewordenen Drogenabhängigen bedeutet dies die Vermittlung in externe, offene Behandlungseinrichtungen. Hier geht es um den Entscheidungsfindungsprozess zur Aufnahme einer langzeittherapeutischen Maßnahme genauso wie um die Vermittlung in ambulante Maßnahmen sowie in Übergangseinrichtungen und Substitutionsprogramme mit psychosozialer Begleitung [53].

Therapiebemühungen konzentrieren sich in der Regel von vornherein auf Überleitungen in freie Therapieeinrichtungen gemäß $\S 35$ Betäubungsmittelgesetz. Hauptsächlicher Inhalt der Beratung durch externe Dienste ist es, inhaltliche Vorbereitungen einzuleiten, d.h. Auseinandersetzung mit dem Konzept der Therapieeinrichtung, Kostenübernahme zu klären, Begleitung in die Therapieeinrichtung, Kooperation mit anstaltsinternen Diensten zu leisten (s. o.). Bei vielen Gefangenen sind allerdings Angst und Vorbehalte gegenüber einer stationären Langzeittherapie tief verwurzelt. Betroffene sehen sich einem Spannungsfeld ausgesetzt, das sich aus der gegenläufigen Praxis von Vertrauensbildung als therapeutischem Prinzip einerseits und Kontrolle und formaler Rückmeldung als Blickrichtung der Justiz andererseits formiert. Weder durch individuelle Anstrengungen Betroffener noch durch qualitativ hochwertige Therapieverfahren lässt sich dieser Widerspruch aufheben. Nach ein- oder mehrmaligem „Durchlauf“ z.T. restriktiver Behandlungsformen und mit längerer Verweildauer in Haft sinkt die Bereitschaft, sich (erneut) auf eine Therapie einzulassen. Ambulante, lebensweltnahe Behandlungsangebote (nach $\S 35$ BTMG) sind immer noch sehr rar und werden zum großen Teil von den Strafvollstreckungskammern auch nicht anerkannt.

Für die stationären Therapieeinrichtungen außerhalb der Gefängnisse entsteht mit der hohen Belegung von Menschen mit justitiellen Therapieauflagen bzw. Menschen, die sich nur in Therapie begeben, um dem Gefängnis zu entkommen, eine problematische Atmosphäre für therapeutische Bemühungen: Die Klienten sind in der Regel wenig therapiemotivierte Menschen, die gefängnis- und/oder drogensubkulturelle Verhaltensweisen einbringen. So können therapeutische Bemühungen oftmals nicht mehr als Anpassungsleistungen an Hausordnungen, TherapeutInnenerwartungen etc. hervorbringen.

Hat der/die Gefangene bereits mehrmals eine solche Therapie durchlaufen, wird sie von den Kostenträgern nicht mehr finanziert. Oftmals wird die Überstellung in die Langzeittherapie jedoch lediglich als Fluchtmöglichkeit genutzt.
Für ihn/sie existiert lediglich die Regelung des $\S 35$ BtMG ff, wonach er/sie aus dem Strafvollzug eine stationäre drogenfreie Langzeittherapie beginnen kann. Und diese Regelung können nur KonsumentInnen illegaler Drogen in Anspruch nehmen.

Die Substitutionsbehandlung im Strafvollzug zielt darauf ab:

- die Nachfrage nach Opiaten im Vollzug zu reduzieren,

- Kriminalität im Vollzug zu reduzieren,

- die Abhängigen gesundheitlich und sozial so zu stabilisieren, dass ihre Motivation, weitergehende Hilfsangebote anzunehmen, gestärkt wird $[54,55]$.

Entsprechend einer angemessenenen, den extramuralen Standards angeglichenen Substitutionsbehandlung im Vollzug sollten folgende Drogenabhängige die Möglichkeit haben, eine Substitutionsbehandlung in Anspruch zu nehmen:

- diejenigen, die auch außerhalb in einer solchen Behandlung waren

- diejenigen, die aus dem Vollzug heraus einen Antrag auf Beginn einer solchen Behandlung stellen und die Bedingungen erfüllen

- diejenigen, die sich in der Phase vor der Entlassung befinden (als vorbereitende Maßnahme etwa drei bis sechs Monate vor der Entlassung)

- Gefangene im Entzug.

Für einen Erfolg müssen Substitutionsbehandlungen nach der Entlassung nahtlos fortgeführt werden. Entscheidende Voraussetzung dafür ist, dass die BUB-Richtlinien für eine kassenärztliche abrechenbare Behandlung Beachtung finden. Entsprechende Vereinbarungen sind bereits während der Haftzeit zu treffen - es muss die Möglichkeit bestehen, die Weiterführung aus der Haft heraus zu organisieren bzw. vorzubereiten.

Psychosoziale Betreuung ist in den Anstalten wie außerhalb eine sinnvolle Ergänzung und wichtig für die Perspektivenentwicklung - entsprechende Angebote etwa zur Arbeits- und Ausbildungsmotivation und -durchführung, zu behandlungsbegleitenden Gesprächsgruppen, Bildung von Betroffenen-Selbsthilfegruppen, Außenorientierungen v. a. auch zu zielgruppenerfahrenen Einrichtungen draußen (z.B. AIDS-Hilfen/JES), soziale Stabilisierungen müssen unterbreitet werden. Dabei muss die psychosoziale Betreuung der jeweiligen individuellen Situation der Gefangenen adäquat sein und entsprechend den unterschiedlichen Bedürfnissen der Gefangenen differenziert werden [56].

Eine genaue Zahl der SubstitutionspatientInnen in Haft liegt für die 16 Bundesländer nicht vor. Schätzungen liegen bei etwa 800 PatientInnen und damit weit unter dem zu erwartenden Bedarf angesichts der hohen Zahlen von Drogenabhängigen im Vollzug. Die Gründe dafür sind vielfältig:

a) Substitutionsbehandlungen werden nicht in allen Bundesländern angeboten (derzeit in sechs).

b) Eine außerhalb des Vollzugs begonnene, ärztlich indizierte Substitutionsbehandlung wird immer noch in vielen Fällen im Gefängnis abgebrochen.

c) Die Substitution ist oftmals begrenzt auf einen Zeitraum von bis zu sechs Monaten (zum Beispiel NRW). 
Die Gründe für eine Substitutionsbehandlung werden mit Beginn der Haft jedoch nicht hinfällig, sondern aus den oben beschriebenen Gründen eher dringender. Die Substitutionsbehandlung ist eine medizinisch begründete Behandlungsform, die unabhängig vom Aufenthaltsort der PatientInnen aufrechtzuerhalten ist: Abbruchgründe ergeben sich dann auch nur aus dem medizinischen und psychosozialen Behandlungskontext und nicht aus den Kontroll- und Sanktionsmaßnahmen des Vollzugs [57]. Diese Behandlungsform ist also keine Sonderbehandlung für Wohlverhalten, kein Beschwichtigungsinstrument für einen „geordneten Vollzug“, sondern eine Krankenbehandlung, die unter den besonderen Bedingungen der Haft den fachlich begründeten Regeln der Substitution außerhalb der Haftanstalten folgen sollte. Die Substitutionsbehandlung in deutschen Gefängnissen ist sehr heterogen. Schwierigkeiten ergeben sich immer wieder, weil die Substitution z.T. innerhalb eines Bundeslandes unterschiedlich gehandhabt wird, aber bei Verlegungen erhebliche Schwierigkeiten von Bundesland zu Bundesland entstehen (vgl. Keppler in diesem Heft). Es fehlen einheitliche Standards, die eine „Best Practice“ beschreiben (wie in England und Schottland „health care standards“; vgl. [13]).

Aufgrund des engen Zusammenlebens einer größeren Zahl von Menschen auf eng umschlossenem Raum gewinnt die Infektionsprophylaxe für die Gefangenen enorm an Bedeutung: Die Unausweichlichkeit des Gefängnisses begrenzt die Schutzmaßnahmen und fördert damit in gewissem Maße die Verbreitung bestimmter Krankheiten (v.a. Infektionen). Für gefangene Drogenabhängige in fast allen deutschen Strafanstalten stehen trotz bekannter erheblicher Ansteckungsrisiken mit den Hepatitiden und HIV - nicht die Schutzmöglichkeiten zur Verfügung, zu denen Drogenabhängige außerhalb des Vollzugs in der Regel einen gewohnt leichten Zugang haben: Sterile Spritzen sind in Freiheit in allen Apotheken und vielen Drogenberatungsstellen zugänglich. Auch diesem Problem wird in den Anstalten mit dem Abstinenzprimat begegnet: Der Verweis auf eine Unterlassung des risikoträchtigen Verhaltens stellt jedoch lediglich einen moralischen Appell dar, der in der Regel an der Realität völlig vorbeigeht, weil er die Lebenswirklichkeit vieler drogenabhängiger Gefangener und die Haftbedingungen ignoriert (vgl. umfassend Nelles/Stöver in diesem Heft).

Harm-reduction-Strategien gehen jedoch über eine Infektionsprophylaxe hinaus: In Freiheit wurden schadensminimierende Ansätze für DrogenkonsumentInnen seit der AIDS-Epidemie Mitte der 80er Jahre entwickelt. Sie gehen von der Prämisse aus, dass Abstinenz nicht für jede/n Drogenabhängigen und nicht für jede/n zu jedem Zeitpunkt ein realistisches oder erstrebenswertes Ziel darstellt. Diejenigen Drogenabhängigen, die vom therapeutischen Ziel der Abstinenz nicht erreicht werden, müssen gleichwohl gesundheitsfördernde Angebote erhalten, um weiteren gesundheitlichen und sozialen Schaden so gering wie möglich zu halten. Hierzu sind hohe existentielle Risiken der Mortalität und Morbidität und sozialen Desintegration zu zählen. Schadensminimierung beinhaltet auch Strategien, die Einstellungen, Haltungen und das Wissen der Zielgruppen zu beeinflussen, damit letztlich eine effektive und zeitstabile Verhaltensänderung in Richtung Risikominderung/-verzicht erfolgen kann $[59,60]$. Im Rahmen der Haft geht es vor allem darum,
- die relativ hohe Rate von Drogentodesfällen während der Haftzeit wie unmittelbar nach Haftentlassung (siehe Beitrag von Heinemann u.a. in diesem Heft) zu reduzieren,

- das Wissen über Übertragungsrisiken zu verbessern

- und das Bewusstsein für Risiken durch gezielte Trainings zu schärfen. Auch die Bediensteten bilden eine Zielgruppe für Aufklärungsarbeit, weil sie als Berufsgruppe starken Infektionsrisiken ausgesetzt sind (Gefährdungspotenziale durch Nadelstichverletzungen bei Haftraumkontrollen).

Kognitiv orientierte Verhaltensänderungsappelle bestimmen nach wie vor die Ansprachen der Zielgruppen. Trainings mit zielgruppenerfahrenen Trainern (Safer-use/Safer-sex-Trainings) bieten dagegen die Chance zu eigenständigem Lernen und zur Verfestigung von Wissen.

Safer-use sollte auch das Ziel von lebensweltnahen und damit glaubwürdigen, mehrsprachig gehaltenen Informations- und Aufklärungsblättern (und anderen Medien) sein, die realistischerweise von einer Fortsetzung des Drogengebrauchs bei vielen Gefangenen nach Haftentlassung ausgehen.

Jacob/Stöver/Keppler [60] fanden, dass die Versorgungslage bezüglich Sexualberatung in den Anstalten insgesamt als unzureichend bezeichnet werden muss [41]. Ein Versorgungsbedarf zu „safer-sex“-Trainings als dauerhaftes Angebot scheint dringend nötig: Es existieren genügend Sexualpraktiken, die Fragen zum Infektionsrisiko aufwerfen. Überdies bieten sich hier Möglichkeiten, Risikobewusstsein zu schärfen, das auch nach der Entlassung in infektionsprophylaktischer Hinsicht wirksam bleiben kann - sowohl für den Bereich der privaten Sexualkontakte als auch in der Beschaffungsprostitution. Werden gelebte sexuelle Orientierungen (Homo- und Heterosexualität) in ihrer Vielfalt nicht durch entsprechende Anerkennung in präventiven Angeboten ernst genommen., wirken diese auf die Zielgruppe unglaubwürdig und realitätsfremd und verlieren an Effektivität.

\section{Perspektiven}

Jenseits zu intensivierender Haftvermeidungsbemühungen [61] erfordern die strukturellen Defizite der medizinischen und psychosozialen Versorgung für Drogenabhängige in Gefängnissen Angleichungsbemühungen der Gesundheitsfürsorge im Vollzug an die Verhältnisse in Freiheit. Außerhalb des Vollzugs setzt sich zunehmend in der Public-Health-Debatte die Erkenntnis durch, nicht nur krankheitsorientiert das individuelle Verhalten, sondern auch die sozialen Verhältnisse und spezifischen Systeme in ihrer Interdependenz und inneren Dynamik auf ihr Gesundheitspotenzial hin zu untersuchen; also nicht nur reaktiv die Krankheit des Einzelnen zu behandeln oder bestenfalls Vorsorge(untersuchungen) und Impfprogramme durchzuführen, sondern darüber hinaus eine aktive Gesundheitsförderung zu betreiben, die alle Akteure in einem bestimmten setting zu integrieren versucht. Erfolgreiche Beispiele für diesen setting-Ansatz in der Gesundheitsförderung sind die von der WHO initiierten Projekte ,Healthy Cities', ,Healthy Schools‘ etc. Dieser Ansatz auf die Gefängnisse übertragen hieße ,Healthy Prisons - der Versuch, die institutionellen Bedingungen mit in die Gesundheitsförderungsdebatte einzubeziehen, statt einseitig maximalistische Forderungen an die Gefangenen zu richten [62]. Dies 
würde auch eine Neuorientierung der Dienste und des Ansatzes der Drogenhilfe im Vollzug bedeuten. Die Kernaussage der Ottawa-Charta, die den setting-orientierten Ansatz theoretisch entwickelt hat, lautet: Menschen sollen befähigt werden, möglichst viel Einfluss auf die Erhaltung und Verbesserung der eigenen Gesundheit auszuüben und Eigenverantwortung für ihre Gesundheit und die anderer zu übernehmen. Dieser Prozess kann aber nicht ohne die Schaffung und Veränderung entsprechender sozialer, politischer und ökonomischer Rahmenbedingungen erfolgen. Auf den Strafvollzug übertragen bedeutet das, das Gefängnis als eine geschlossene, räumliche Einheit mit bestimmten Regeln zu betrachten, in der auf mehreren Ebenen verschiedene Akteure mit unterschiedlichen Interessen und jeweils spezifischen Lebens- und Arbeitswelten aufeinandertreffen. Unter einem Gesundheitsförderungsaspekt darf die Verantwortung für Gesundheit nicht einseitig an betroffene Gefangene delegiert werden, ohne die gesundheitsabträglichen Lebensbedingungen im Vollzug zu thematisieren. Die anderen Gefangenen, aber auch Bedienstete und die physischen, zeitlichen und organisatorischen Bedingungen sind mit in die Überlegungen einer Gesundheitsförderung im Gefängnis einzubeziehen. Schließlich geht es darum, die Institution Gefängnis als ein System unter vielen zu begreifen und Verbindungen mit anderen Institutionen herzustellen (kommunale/regionale Hilfeorganisationen, Familie, Arbeit etc.).

\section{Literatur}

${ }^{1}$ Boetticher A, Stöver H. Gesundheitsfürsorge $§ \S 56-66$ StVollzG. In: Feest J (Hrsg). Kommentar zum Strafvollzugsgesetz. Neuwied, Kriftel: Luchterhand 2000; $366-428$

2 Preusker H. Suchtprobleme im Justizvollzug. In: DHS, Gassmann R (Hrsg). Suchtprobleme hinter Mauern. Freiburg: Lambertus; 123 - 130 ${ }^{3}$ Kreuzer A. Bedingungen der strafrechtlichen Praxis in stationären Einrichtungen. In: DHS, Gassmann R (Hrsg). Suchtprobleme hinter Mauern. Freiburg: Lambertus; 35-64

${ }^{4}$ Justizministerium des Landes NRW. Protokoll über die Drogentagung am 19.9.1996 in der Justizakademie des Landes NRW in Recklinghausen. Düsseldorf

${ }^{5}$ Dolde G. Therapie in Untersuchungs- und Strafhaft. In: DHS, Gassmann R (Hrsg). Suchtprobleme hinter Mauern. Freiburg: Lambertus; $131-144$

${ }^{6}$ Maelicke H. Ist Frauenstrafvollzug Männersache? Eine kritische Bestandsaufnahme des Frauenvollzuges in den Ländern der Bundesrepublik Deutschland. Baden-Baden 1995

${ }^{7}$ Meyenberg R, Stöver H, Jacob J, Pospeschill M. Infektionsprophylaxe im Justizvollzug - Abschlußbericht. Oldenburg: BIS-Verlag 1999

${ }^{8}$ Küfner H. Evaluation von externen Beratungsangeboten für suchtgefährdete und suchtkranke Gefangen in bayerischen Justizvollzugsanstalten. Sucht 1998; 44, H.6: 406-408

${ }^{9}$ Küfner H, Beloch E. Externe Beratung für Gefangene mit Drogenproblemen in bayrischen Justizvollzugs. In: Jacob J, Keppler K, Stöver H (Hrsg). LebHaft - Gesundheitsförderung für Drogen Gebrauchende im Strafvollzug: AIDS-Forum 2001; Bd. 42, Teil 1 u. 2

${ }^{10}$ Koch U, Ehrenberg S. Akzeptanz AIDS-präventiver Botschaften: Evaluation der Aufklärungs- und Beratungsarbeit bei i.v. Drogenabhängigen in der Bundesrepublik Deutschland. In: Deutsche AIDSHilfe e.V (Hrsg). AIDS und Drogen II. Evaluation AIDS-präventiver Botschaften. Berlin: AIDS-Forum D.A.H 1992; Bd. IX: 27 -101

${ }^{11}$ Kleiber D, Pant A. HIV-Needle-Sharing-Sex. Eine sozialepidemiologische Studie zur Analyse der HIV-Prävalenz und riskanter Verhaltensweisen bei i.v. Drogenkonsumenten. Band 69a Schriftenreihe des Bundesministeriums für Gesundheit. Baden-Baden: Nomos 1996

${ }^{12}$ AMSEL-Forschungsprojekt, Projektgruppe Rauschmittelfragen. Abschlußbericht Bd. 1 Jugendberatung und Jugendhilfe, Frankfurt: (Selbstverlag) 1991
${ }^{13}$ Stöver H. Drug and HIV/AIDS Services in European Prisons. Schriftenreihe „Gesundheitsförderung im Justizvollzug“, Bd. 8. Oldenburg: BISVerlag 2002

${ }^{14}$ Gore S, Bird A, Ross A. Prison Rites: Starting to inject inside. British Medical Journal 1995; 311: 1135-1136

${ }^{15}$ European Network on HIV/AIDS and Hepatitis Prevention in Prison Annual Report to the European Commission. Bonn/WIAD: Selbstverlag May 1998

${ }^{16}$ Ingle R. 20\% of Prisoners Claim Drug Use Began in Custody In: Irish Times (Hrsg).. Ireland Sunday, 22 Aug. 1999

17 Edgar K, O'Donell I. Mandatory Drug Testing in Prisons - An Evaluation. Home Office Research And Statistics Directorate, Research Findings No. 75/1998, London

${ }^{18}$ Kingma S, Goos C. Drugs and AIDS in Prisons in Europe: A Perspective from UNAIDS and WHO. In: Report of the 3rd European Conference on Drug and HIV/AIDS Services in Prison, Amsterdam Febr. 1997; 3 - 5

${ }^{19}$ Turnbull PJ, Webster R. Demand Reduction Activities in the Criminal Justice System in the European Union. Final Report. The Centre for Research on Drugs and Health Behaviour, (Bezug: EMCDDA - Lissabon). London August 1997

${ }^{20}$ UNAIDS. Trends in HIV incidence and prevalence: natural course of the epidemic or results of behavioural change? UNAIDS Best Practice Collection - Key Material. Geneva/Switzerland 1999

${ }^{21}$ WHO. Health In Prisons. A European Network For Promoting Health in Prisons (o. J.).

22 Weilandt C. Prävalenz HIV-bezogenen Risikoverhaltens im Justizvollzug - Ergebnisse einer Machbarkeitsstudie. Infektionsepidemiologische Forschung des RKI. Berlin 1997; H. II/: 27 - 34

${ }^{23}$ Weild A, Curran L, Parry J, Bennet D, Newham J, Gill ON. The prevalence of HIV and associated risk factors in prisoners in England and Wales in 1997: Results of a national survey. Abstract (abstract 23510) presented on the 12th World AIDS Conference in Geneva, 28 June-3rd July. 1998

24 Wedershoven C. Katamnesen der HIV-Infektion bei drogenabhängigen und nicht-drogenabhängigen Inhaftierten im Vergleich im Justizvollzug des Landes Nordrhein-Westfalen. 1998

25 Jacob J, Keppler K, Stöver H (Hrsg). LebHaft: Gesundheitsförderung für Drogen Gebrauchende im Strafvollzug. AIDS-Forum. 2001; Bd. 42, Teil 1 u. 2

${ }^{26}$ Knapp R. AIDS im Strafvollzug. Zur Situation HIV-Infizierter und AIDSKranker Strafgefangener unter besonderer Berücksichtigung der Problematik intramuralen Drogenkonsums: Ergebnisse einer empirischen Erhebung und rechtliche Konsequenzen (Unveröff. Diss.). Bonn 1996

${ }^{27}$ Heudtlass JH, Stöver H (Hrsg). Risiko mindern. Frankfurt: Fachhochschulverlag 2000

${ }^{28}$ Bornemann R, Stöver H. Schadensminimierung. In: Fengler J (Hrsg). Handbuch der Suchtbehandlung - Beratung, Therapie, Prävention. Landsberg/Lech: ecomed 2002; 468-477

${ }^{29}$ Keppler KH, Nolte F, Stöver H. Übertragungen von Infektionskrankheiten im Strafvollzug - Ergebnisse einer Untersuchung in der JVA für Frauen in Vechta. Sucht 1996; H. 96 (2): 98 - 107

30 Outbreak of HIV in a Scottish Prison: Why Did It Happen? In: Canadian HIV/AIDS Policy \& Law Newsletter. 1996; 2 (3): 13-14

${ }^{31}$ Staib W. Sexualität im Gefängnis - Einblicke in das Leben inhaftierte Männer. Janus-Gefangenenzeitung der VA. Freiburg 17. Jg., H. 1/1990; $15 \mathrm{ff}$

32 Schwermer L. HIV/AIDS. Gesundheitsamt Gesundheitsbericht der Stadt Essen: (Selbstverlag). Essen 1996; Bd. V

33 Bundesamt für Statistik der Schweiz. Die Gesundheit der Insassen in Schweizer Gefängnissen 1993. Resultate der Schweizerischen Gesundheitsbefragung 1992/93. Bern: Selbstverlag Bundesamt f. Statistik 1998; (Bd. 14)

${ }^{34}$ Gaube J, Feucht HH, Laufs R, Polywka S, Fingscheidt E, Müller HE. Hepatitis $A, B$ und $C$ als desmoterische Infektionen. Gesundheitswesen 1993; 55 (5): $246-249$

35 Heinemann A, Püschel K. Pilotprojekt zur Infektionsprophylaxe für Drogenabhängige in der Anstalt des offenen Vollzugs in Vierlande (Anstalt XII) - Medizinische Begleitforschung. Institut für Rechtsmedizin. Eppendorf: Universitätskrankenhaus 1998

${ }^{36}$ Stark K, Müller R, Bienzle U, Guggenmoos-Holzmann I. Frontloading: a risk factor for HIV and hepatitis $\mathrm{C}$ virus among injecting drug users in Berlin. AIDS 1996; 10: $311-317$

${ }^{37}$ Holsen DS, Harthug S, Myrmel H. Prevalence of Antibodies to Hepatitis $C$ Virus and Association with Intravenous Drug Abuse and Tattooing in a national Prison in Norway. Eur. Clin. Microbiol. Infect. Dis. 1993; 9: $673-676$ 
${ }^{38}$ Gbordzoe E. Bewertung der HIV- und Hepatitis-Prävalenz im badenwürttembergischen Justizvollzug. ZfStrVo 1997; 2: 87-89

39 Breedek UF, Iselborn M, Altmann D, Rex R. HIV und AIDS in Berliner Justizvollzugsanstalten. InfFo 1997; 1: 13-21

${ }^{40}$ Goll G. Stellungnahme des Justizministeriums zur Frage „Spritzenvergabe in den Justizvollzugsanstalten“ v. 30.1.97. Der Vollzugsdienst. $3 / 97 ; 8 \mathrm{f}$

${ }^{41}$ BMG/BMJ. Dokumentation der Expertenanhörung zu dem Thema Drogen und HIV-Prävention im Justizvollzug am 17./18. August 1994 in Bonn. Bonn 22.2.1995

42 Koops A, Thiele B, Heinemann A, Püschel K. Prävalenz viraler Infektionskrankheiten (HIV, Hepatitis $B+C$ ) bei Inhaftierten im Hamburger Justizvollzug im Vergleich mit i.v. Drogenabhängigen in anderen Einrichtungen. Vortrag auf dem „2. Europäischen Seminar zu HIV und Hepatitis im Justizvollzug“. 12./13.12.97 Bonn

${ }^{43}$ Stöver H, Weilandt C. Prävalenz viraler Infektionskrankheiten und infektionsrelevanten Risikoverhaltens im deutschen Justizvollzug. In: Infektionsepidemiologische Forschung. H. 2/1997

${ }^{44}$ Stöver H. Die Verbreitung viraler Infektionskrankheiten. In: Jäger $\mathrm{H}$ (Hrsg). AIDS - Herausforderungen für Forschung, Behandlung und das Leben mit HIV. Landsberg/Lech: mi 2000; 520-529

${ }^{45}$ Stöver H. Drug Substitution Treatment and Needle Exchange Programs in German and European Prisons. Journal of Drug Issues 2002; $573-596$

${ }^{46}$ Stöver H. Virale Infektionskrankheiten und Prophylaxeansätze im Justizvollzug. In: Brockmeyer NH, Brodt R, Hoffmann K, Reimann G, Stücker M, Aaltmeyer P (Hrsg). HIV-Infekt. Berlin u.a: Springer 2000; $835-845$

${ }^{47}$ Thiel A. Infektionsprophylaxe in der JVA Vierlande - Zur Abgabe steriler Einwegspritzen in einem Hamburger Modellprojekt. In: Jacob J, Keppler K, Stöver H (Hrsg). Zum Umgang mit Drogengebrauch und Infektionsgeschehen (HIV/AIDS und Hepatitis) im Strafvollzug. Berlin: Selbstverlag 1997; 159-166

${ }^{48}$ Alm-Merk H. Staatliche Spritzenvergabe als Beitrag zur Resozialisierung von GefängnisinsassInnen In: Meyenberg R (Hrsg). Neue Wege in der Drogenpolitik/Drogenhilfe. Oldenburg: BIS-Verlag 1998; 19- 28

${ }^{49}$ Stöver H. Peer Support als methodische Strategie des Einbezugs von Betroffenenkompetenz in die Drogenhilfe. In: Böllinger L, Stöver H (Hrsg). Drogenpraxis, Drogenrecht, Drogenpolitik. Handbuch für Drogenbenutzer, Eltern, Drogenberater, Ärzte und Juristen. Frankfurt: Fachhochschulverlag 2002; 378 - 385
${ }^{50}$ Jacob J, Stöver H. Drogenabhängigkeit und Strafvollzug. In: Gölz J (Hrsg). Der drogenabhängige Patient: Urban u. Fischer 1999; 2 Aufl.: $172-190$

${ }^{51}$ Behrendt K, Degkwitz P, Trüg E. Schnittstelle Drogenentzug - Strategien, Praxis und Perspektiven vor dem Hintergrund des Paradigmenwechsels in der Drogenhilfe. Freiburg i. Br: Lambertus 1995

52 Dreger L. Interne Beratung und Betreuung Drogenabhängiger in Justizvollzugsanstalten am Beispiel NRW. In: DHS, Gassmann R (Hrsg). Suchtprobleme hinter Mauern. Freiburg: Lambertus; 167-174

${ }^{53}$ Kunkel-Kleinsorge S. Externe Drogenberatung - Ein Praxisbericht. In: DHS, Gassmann R (Hrsg). Suchtprobleme hinter Mauern. Freiburg: Lambertus; $175-184$

54 Justizminister Schleswig-Holstein. Suchthilfekonzept für den Justizvollzug des Landes Schleswig-Holstein (Ms.) 1995

${ }^{55}$ Keppler K. Methadonsubstitution im Gefängnis. In: Jäger H (Hrsg) AIDS - Herausforderungen für Forschung, Behandlung und das Leben mit HIV. Landsberg/Lech: mi 2000; 530-534

${ }^{56}$ Schaper G. Praxis, Probleme und Grenzen der psychosozialen Begleitung der Substitutionsbehandlung im Strafvollzug. Zum Umgang mit Drogengebrauch und Infektionsgeschehen (HIV/AIDS und Hepatitis) im Strafvollzug. Berlin: Selbstverlag 1997; $101-108$

${ }^{57}$ Kommission zur Entwicklung eines umsetzungsorientierten Drogenkonzeptes für den Hamburger Strafvollzug. Abschlußbericht der vom Justizsenator der Freien und Hansestadt eingesetzten Kommission. Hamburg (Ms.). Febr. 1995

58 Jacob J, Stöver H (Hrsg). Minimierung gesundheitlicher Risiken bei Drogenkonsum unter Haftbedingungen. Ein methodisch-didaktisches Arbeitsbuch für die Praxis im Strafvollzug. Oldenburg: BIS-Verlag 1998

${ }^{59}$ Stöver H, Trautmann F (Hrsg). Risk Reduction For Drug Users in Prisons. Utrecht: Trimbos Institute 2001

${ }^{60}$ Jacob J, Keppler K, Stöver H. Zum Umgang mit Drogengebrauch und Infektionsgeschehen (HIV/AIDS und Hepatitis) im Strafvollzug Berlin: Deutsche AIDS-Hilfe-Selbstverlag 1997

${ }^{61}$ Keppler K. Strategien der Haftvermeidung und der Wert der ärztlichen Bescheinigungen. In: Jäger H (Hrsg). AIDS - Neue Perspektiven - Therapeutische Erwartungen - Die Realität 1997. Landsberg/Lech: ecomed 2000; 488-490

62 Stöver H. Healthy Prisons: Strategien der Gesundheitsförderung im Justizvollzug. Oldenburg: BIS-Verlag 2000 